\title{
Tuning inner-layer oxygen functional groups of reduced graphene oxide by potentiostatic oxidation for high performance electrochemical energy storage devices
}

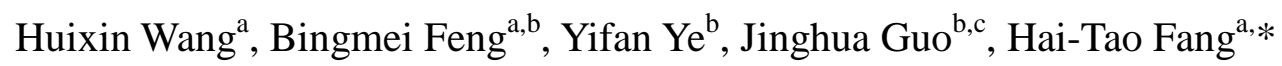 \\ ${ }^{a}$ School of Materials Science and Engineering, Harbin Institute of Technology, Harbin \\ 150001, China. \\ ${ }^{b}$ Advanced Light Source, Lawrence Berkeley National Laboratory, Berkeley, California \\ 94720, United States. \\ ${ }^{c}$ Department of Chemistry and Biochemistry, University of California, Santa Cruz, \\ California 95064, United States. \\ * Corresponding author. E-mail address: htfang@hit.edu.cn (H.T. Fang)
}

Keywords Graphene; Energy storage; Lithium ion; Bulk diffusion; Potentiostatic oxidation

\begin{abstract}
The electrochemical lithiation/delithiation of oxygen-containing functional groups (OCFGs) of nanocarbon materials, particularly graphene, have attracted intensive interest in recent years. Here, we propose a controllable potentiostatic oxidation approach to tune the OCFGs of as-prepared reduced graphene oxide ( $\mathrm{rGO}$ ) in a carbonate-based electrolyte to improve the specific capacity and rate capability. By X-Ray absorption spectroscopy in total fluorescence yield mode and X-Ray diffraction, we confirm that potentiostatic oxidations generate new OCFGs in the inner-layer of rGO. The content of OCFGs increases as oxidation potential being elevated. Such increasing of OCFGs in quantity significantly enhances the capacity. For instance, the specific capacity of $170.4 \mathrm{mAh} \mathrm{g}^{-1}$ for pristine $\mathrm{rGO}$ electrode is increased to $290.5 \mathrm{mAh} \mathrm{g}^{-1}$ after the oxidation at 5.0 V. We demonstrate that oxidations at moderate potentials can reduce the electrochemical and ohmic polarizations of rGO electrodes without
\end{abstract}


deteriorating diffusion dynamic, thereby improving rate capability. After the optimal oxidation at $4.7 \mathrm{~V}$, rGO electrode exhibits an excellent rate capability, delivering $58.4 \mathrm{mAh}$ $\mathrm{g}^{-1}$ at $20 \mathrm{~A} \mathrm{~g}^{-1}$.

\section{Introduction}

Carbon materials play a crucial role in electrochemical energy storage devices. The electrochemical lithiation/delithiation of oxygen-containing functional groups (OCFGs), i.e. $\mathrm{C}-\mathrm{O}-\mathrm{C},>\mathrm{C}=\mathrm{O}$ and $\mathrm{COOH}$, of carbon nanotube (CNT) [1-6], graphene[7-19], CNT/graphene hybrid [20, 21] and CNT/carbon sphere hybrid [22] in $1.5 \sim 4.5 \mathrm{~V}\left(v s \mathrm{Li} / \mathrm{Li}^{+}\right.$) have been attracted intensive interest in recent years. The involved faradic processes contribute to additional charge storage, thus enhancing the energy density of these nanocarbon materials. A pioneering study by Lee et al. demonstrated the lithiation/delithiation redox reactions of OCFGs on CNTs in a carbonate-based electrolyte improve the capacity significantly [1]. Jang et al. figured out that these redox reactions are present in reduced graphene oxide (rGO) as well [7]. Taking advantage of pseudocapacitive behavior in a wide potential range for the lithiation/delithiation of the OCFGs, and the unique features of graphene including 2D geometry, controllable surface chemical structure, exceptional mechanical and electric properties, eco-friendly cells with graphene as both cathode and anode materials, named all-graphene-batteries [7, 13] or all-graphene-lithium ion supercapacitors [12, 18], have been explored. An all-graphene-cell delivers an energy density of $225 \mathrm{Wh} \mathrm{kg}^{-1}$ [13], 40 times higher than that of conventional supercapacitors.

It is reported that more OCFGs could contribute to higher capacity [11, 20]. Excessive OCFGs, however, may severely interrupt the $\pi$ conjugation, which is vital for the in-plane electronic transport through delocalized electrons. If the pursuit of OCFGs enrichment is overdone, the lowered electronic conductivity will eventually deteriorate the electrochemical performance. Therefore, it is necessary to optimize the number of OCFGs to address the tradeoff between more active OCFGs and higher electronic conductivity. To achieve the rGO electrode with wonderful electrochemical properties, such as specific capacity and rate 
capability, the chemical engineering on its OCFGs is imperative. Approaches by adjusting oxidizing agent amount in the stage of preparing graphene oxide (GO) [11], or changing thermal reduction temperatures [10, 15], electrochemical reduction potential [23] and hydrothermal reduction duration [14], realize controlling the content of oxygen in rGO. In general, these approaches are confined in the period of rGO fabrication. To broaden the way to tune the OCFGs of rGO precisely, a new strategy through a post-modification on rGO is worth of attention.

The precursor of $\mathrm{rGO}$, namely $\mathrm{GO}$, is fabricated by wet-chemistry processes involving harsh oxidation, resulting in very close $\mathrm{C} / \mathrm{O}$ ratio for each $\mathrm{GO}$ sheets. Graphene sheets are prone to restacking into multi-layer structure due to the strong $\pi-\pi$ interaction among them. As from the reduction of GO, rGO contains OCFG residual both on the outmost surface and in the inner layers of the restacked multi-layer architecture. OCFGs in the inner layers can take part in electrochemical lithiation/delithiation reactions through the lithium ion diffusion, which will even become the main way to store charge when the layer number is large. Therefore, it is greatly desired that the new post-modification approach has the capability to tune the OCFGs not only on the surface, but also in the inner layers of rGO.

Herein, we develop a controllable potentiostatic oxidation approach to tune the content of OCFGs for as-prepared rGO. X-Ray absorption spectroscopy in bulk-sensitive total fluorescence yield mode (TFY-XAS), X-Ray diffraction (XRD) and Raman spectroscopy were employed to characterize the modified rGO electrodes aimed at the confirmation of bulk-tuning for OCFGs. We demonstrated that this approach enhances the specific capacity of rGO electrode. In addition, the rate capability could also be significantly improved by optimizing the oxidation potential.

\section{Experimental}

\subsection{Electrode preparation and cell assembly}

The rGO powder (supplied by Advanced Carbons Division of Shenyang National 
Laboratory for Materials Science) used in this study was produced from graphite oxide via a common thermal reduction route. Fig. S1 shows scanning electron microscopy (SEM) and transmission electron microscopy (TEM) images, nitrogen adsorption/desorption isotherms and X-Ray photoelectron spectroscopy (XPS) spectrum of the rGO powder. The Brunauer-Emmett-Teller specific surface area of rGO is $334 \mathrm{~m}^{2} \mathrm{~g}^{-1}$, indicating its 8-layer structure in average. Its carbon/oxygen atomic ratio is 9.5:1. To prepare rGO electrodes, a slurry was first prepared by mixing $70 \mathrm{wt} \%$ of rGO powder with $20 \mathrm{wt} \%$ conductive carbon black (Super P) and 10wt\% Poly (vinylidene fluoride) (PVDF) dissolved in N-methyl-2-pyrrolidone (NMP). The slurry was further diluted by NMP to a moderate viscosity, then magnetically stirred for more than 12 hours and casted onto an Al foil via a tape casting process. After drying at $50{ }^{\circ} \mathrm{C}$ in an oven for 6 hours, the $\mathrm{rGO}$-coated $\mathrm{Al}$ foil was punched to obtain plates with diameter of $15.5 \mathrm{~mm}$. Then these plates were pressed under 60 $\mathrm{MPa}$ and dried in vacuum at $120{ }^{\circ} \mathrm{C}$ for 12 hours to obtain rGO electrodes. The loading mass of rGO in electrodes $(\sim 0.15 \mathrm{mg})$ are listed in Table $\mathrm{S} 1$.

In an argon filled glove box with oxygen and water less than 1ppm (MBRAUN, Germany), an rGO electrode and a Li metal foil separated by Celgard M824 membrane were assembled into a CR-2032 type coin cell. $1 \mathrm{M} \mathrm{LiPF}_{6}$ in ethylene carbonate (EC)/dimethyl carbonate (DMC) (1:1 vol) solution was used as the electrolyte.

\subsection{Potentiostatic oxidation and electrochemical measurements}

The rGO/Li CR-2032 coin cells were adopted to perform potentiostatic oxidations as illustrated in Fig. 1. After galvanostatic charging at $50 \mathrm{~mA} \mathrm{~g}^{-1}$ to high potentials ranging from 4.6 to $5.0 \mathrm{~V}$, oxidization for $10 \mathrm{~h}$ was carried out. $\mathrm{rGO}$ electrodes were labeled according to oxidation potentials and time.

The specific capacities of rGO electrodes were evaluated by galvanostatic charge-discharge measurements. Charge-discharge cycles were conducted between 1.5 to 4.5 $\mathrm{V}$ (vs $\mathrm{Li} / \mathrm{Li}^{+}$). A galvanostatic battery tester (LANHE CT2001A, China) and an electrochemical workstation (Autolab 302N, Netherland) with a faster data acquisition rate of 
780 samples per second were used for measuring capacities at low and high current densities, respectively. The values of current densities and capacities in this paper is based on the mass of graphene in electrodes.

Galvanostatic intermittent titration (GITT) measurements for pristine and oxidized rGO electrodes after 10 charging/discharging cycles were carried out with the LAND CT2001A. A constant current pulse of $50 \mathrm{~mA} \mathrm{~g}^{-1}$ was applied for 10 minutes, followed by an open-circuit relaxation period of $2 \mathrm{~h}$. Potentials at end of each open-circuit relaxation were recorded to calculate $d E / d x$, the slope of GITT curve at each capacity $(x)$ value.

Electrochemical impedance spectroscopy (EIS) measurements at various potentials for pristine and oxidized rGO electrodes after 10 charging/discharging cycles were performed with frequency response analyzer (Autolab FRA32 module embedded in Autolab 302N). The frequency range was from $10 \mathrm{mHz}$ to $1 \mathrm{MHz}$ with an ac signal amplitude of $10 \mathrm{mV}$. Impedance spectra were analyzed using NOVA software to obtain charge transfer resistance $\left(\mathrm{R}_{\mathrm{CT}}\right)$ and solid-electrolyte interphase (SEI) resistance $\left(\mathrm{R}_{\mathrm{SEI}}\right)$. In addition, the lithium ion diffusion coefficients are measured by EIS method, specifically calculated by following equation $[24,25]$ :

$$
D=\frac{1}{2}\left[\frac{V_{m}}{F A \sigma}\left(-\frac{d E}{d x}\right)\right]^{2}
$$

where $D$ is diffusion coefficient of ion, $A$ is apparent area of electrode, $\sigma$ is Warburg coefficient, $V_{m}$ is molar volume of active material, $F$ stands for the Faraday constant (96480 $\left.\mathrm{C} \mathrm{mol}^{-1}\right)$. Here, A is $1.886 \mathrm{~cm}^{2} . V_{m}$ value for each $\mathrm{rGO}$ is calculated by $V_{m}=(\mathrm{d}(002) / 0.336$ $\mathrm{nm}) \times 5.31 \mathrm{~cm}^{3} \mathrm{~mol}^{-1}$, in which $\mathrm{d}(002)$ is interlayer spacing of rGO measured by XRD, 0.336 $\mathrm{nm}$ and $5.31 \mathrm{~cm}^{3} \mathrm{~mol}^{-1}$ are the interlayer spacing and molar volume of graphite with a density of $2.26 \mathrm{~g} \mathrm{~cm}^{-3}$, respectively. Warburg coefficient is calculated from real part of impedance, Z', by linear fitting on $Z^{\prime}-\omega^{-1 / 2}$ plot, where $\omega$ is angle frequency.

\subsection{Structure and Chemistry Characterizations}

After potentiostatic oxidations, the cells were disassembled to preform characterizations 
of rGO electrodes. The interlayer spacing of rGO was measured by an X-Ray diffractometer (Bruker D8 advance, German) using $\mathrm{Cu} \mathrm{K} \alpha$ radiation with $0.04^{\circ}$ interval step. A confocal microscope Raman spectrometer system (BWTEK BWS435-532SY, America) with $532 \mathrm{~nm}$ excitation wavelength was used to record Raman spectra. The laser power was as low as 50 $\mathrm{mW}$.

Carbon K-edge XAS of pristine and oxidized rGOs were acquired at BL8.0.1 in the Advanced Light Source, Lawrence Berkeley National Laboratory. In order to eliminate interference from carbon species in PVDF binder, binder-free rGO electrodes were prepared by tightly compacting rGO powder with stainless steel grids (400 mesh). Two binder-free rGO electrodes were oxidized at 4.7 and $5.0 \mathrm{~V}$, respectively, for $10 \mathrm{~h}$ in CR-2032 coin cells before the XAS measurement and one pristine rGO electrode (charged to $4.5 \mathrm{~V}$, pris-4.5V) in fully-delithiated state was also prepared as control sample.

\section{Results and discussion}

\subsection{Characterization of potentiostaticly oxidized $r G O$ electrodes}

After potentiostatic oxidations at different potentials, the content of OCFGs increases and the interlayer spacing gets to be enlarged as illustrated in Fig. 1. Details on the chemical and structural changes by the oxidations are described and discussed below.

TFY-XAS is a powerful technique to probe the unoccupied states of molecules. In comparison with extremely surface-sensitive XPS, TFY-XAS has the advantage of larger detection depth of approximate one hundred nanometers, thereby minimizing the interference from $\mathrm{C}$ species in the solid-electrolyte interphase (SEI) layer on electrode surface. To investigate the variation trend of each OCFG in rGO after potentiostatic oxidations at different potentials, the carbon K-edge XAS of representative electrodes including pris-4.5V, 4.7V_10h and 5.0V_10h are analyzed as shown in Fig. 2. Peak B at $285.2 \mathrm{eV}$ corresponds to the transition of $\mathrm{C} 1 \mathrm{~s}$ to $\pi^{*}$ orbitals in $\mathrm{C}=\mathrm{C}$ bond, a typical signature of the $\mathrm{sp}^{2}$ hybridized carbon materials. Peak I at $292.5 \mathrm{eV}$ arises from the 1 s-to- $\sigma^{*}$ transition in $\pi$-conjugated 
carbon materials. It is worthwhile to mention that the peak $\mathrm{H}$ at $291.8 \mathrm{eV}$ is the core-excitation $[26,27]$ and its presence indicates that there is still large $\mathrm{sp}^{2}$ network domain exiting in the rGO electrodes after potentiostatic oxidations. Originating from the interruption of $\mathrm{sp}^{2}$ network domain by OCFGs, the defect-induced peak $\mathrm{A}$ at $283.7 \mathrm{eV}$ [28, 29] is identified after the deconvolution. The C, D, E and F peaks at 286.5, 287.8, 288.7 and 289.7 $\mathrm{eV}$ are from $\mathrm{C} 1 \mathrm{~s}$ to $\pi^{*}$ resonances of $\mathrm{C}-\mathrm{OH}, \mathrm{C}-\mathrm{O}-\mathrm{C},>\mathrm{C}=\mathrm{O}$ and $\mathrm{COOH}$, respectively [30, 31]. Besides, the peak $\mathrm{G}$ at $290.3 \mathrm{eV}$ is attributed to carbonates, such as $\mathrm{Li}_{2} \mathrm{CO}_{3}$ and $\mathrm{ROCOOR}$ [32-34], in SEI layers formed on rGO electrodes. $\mathrm{J}_{1}-\mathrm{J}_{5}$ peaks from $\mathrm{C} 1 \mathrm{~s}$ to $\sigma^{*}$ resonances of OCFGs bonds are also present in higher photon energy region [35].

The area ratios of A, C, D, E, F and G peaks with respect to B peak, quasi-quantitively proportional to the relative concentration of each species in rGO electrodes, are plotted in Fig. 2(b). Except a maximum of $>\mathrm{C}=\mathrm{O}$ is obtained for $4.7 \mathrm{~V} \_10 \mathrm{~h}$ electrode, the total concentration of OCFGs (i.e. $\mathrm{C}-\mathrm{OH}, \mathrm{C}-\mathrm{O}-\mathrm{C},>\mathrm{C}=\mathrm{O}$ and $\mathrm{COOH}$ ) is favorable to be increased with elevating the oxidation potential. The increase of defect (peak A) indicates the break of $\mathrm{C}_{6}$ rings providing the reaction sites for oxygen interaction. This result suggests that potentiostatic oxidation is able to facilitate the generation of new OCFGs in carbonate-based electrolytes. It has been reported that the $\mathrm{sp}^{2}$ conjugative carbon structures of graphite or graphene sheets can be destructed and transformed into OCFGs under high oxidation potentials in aqueous solutions [30, 36, 37] or ionic liquids [38, 39]. But electrochemical oxidations in carbonate-based electrolytes are still rarely reported. We expect such potentiostatic oxidation approach to be a controllable way for improving the pseudocapacitive performance of rGO electrode.

XRD patterns in Fig. 2(c) shows a shift of (002) peak from $25.00^{\circ}$ towards $22.29^{\circ}$ as oxidation potentials increasing to $5.0 \mathrm{~V}$, corresponding to an enlargement of interlayer spacing from $3.56 \AA$ to $3.99 \AA$ A. Fig. 2(d) indicates that there is no significant change for $G$ band in Raman spectra. Although the intercalation of anions at high potentials is readily to enlarge the interlayer spacing [40,41], such intercalation could be excluded due to the absence of the essential splitting of Raman G band for graphite intercalated compounds 
[42-44]. We attribute this interlayer spacing enlargement to the enhanced repulsion among polar OCFGs in the interior of the rGO rather than the intercalation of $\mathrm{PF}_{6}^{-}$. The enlargement of interlayer spacing also confirms that tuning inner-layer OCFGs in rGO is achieved by our potentiostatic oxidation approach.

\subsection{Effect of oxidation potentials on the electrochemical performance}

OCFGs (i.e. C-O-C [9], >C=O [7] and $\mathrm{COOH}[1,2,20]$ ) have been shown to be electrochemically active in the carbonate-based electrolyte between $1.5 \sim 4.5 \mathrm{~V}$, while $\mathrm{C}-\mathrm{OH}$ might be less active as its lithiation potential as low as $0.585 \mathrm{~V}$ predicted by a first-principle calculation[45]. Fig. 3(a) shows galvanostatic charge-discharge curves (at $50 \mathrm{~mA} \mathrm{~g}^{-1}$ ) with varied slopes against potentials, indicating that the charging-discharging of all electrodes involves pseudocapacitive reactions from active OCFGs. The specific capacity increases from 177.5 $\mathrm{mAh} \mathrm{g}^{-1}$ to $290.5 \mathrm{mAh} \mathrm{g}^{-1}$ as oxidation potentials rise from $4.6 \mathrm{~V}$ to $5.0 \mathrm{~V}$, higher than 170.4 $\mathrm{mAh} \mathrm{g}^{-1}$ for pristine rGO electrode. Potentiostatic oxidations successfully induced more electrochemically active OCFGs into rGO electrodes to act as pseudocapacitive reaction sites in the carbonate-based electrolyte.

These oxidized rGO electrodes exhibit good cycle performance as shown in Fig. 3(b). During the long-time operation, pristine and $4.6 \sim 4.8 \mathrm{~V}$ oxidized electrodes even show a self-enhanced cycle performance, which is likely due to the gradual penetration of electrolyte into more inner pores of electrodes as described in several published literatures [11, 13-16]. In contrast, the 4.9 and 5.0 $\mathrm{V}$ oxidized electrodes suffer a slight fading because of the lack of a stable SEI layer [17].

The rate performance of these oxidized rGO electrodes is shown in Fig. 3(c). When the current density is set below $2 \mathrm{~A} \mathrm{~g}^{-1}$, the specific capacity shows an upward tendency as oxidation potentials increasing. Whereas under the higher current density, the rGO electrodes oxidized at moderate potentials deliver higher capacities than others. The insert in Fig. 3(c) shows the normalized capacities at $20 \mathrm{~A} \mathrm{~g}^{-1}$ by those at $50 \mathrm{~mA} \mathrm{~g}^{-1}$. In comparison with pristine 
rGO electrode, moderately oxidized electrodes $\left(4.6 \mathrm{~V} \_10 \mathrm{~h}, 4.7 \mathrm{~V} \_10 \mathrm{~h}\right.$ and $4.8 \mathrm{~V} \_\mathrm{h}$ electrodes $)$ offer higher normalized capacities $(33.4 \%, 29.2 \%$ and $25.0 \%$, respectively). The 4.9V_10h electrode has a normalized capacity close to pristine electrode at $20 \mathrm{~A} \mathrm{~g}^{-1}$, while this capacity falls to only $0.4 \%$ for $5.0 \mathrm{~V} \_10 \mathrm{~h}$. If considering real application, we expect the $4.7 \mathrm{~V} \_10 \mathrm{~h}$ electrode (58.4 $\mathrm{mAh} \mathrm{g}^{-1}$ at $20 \mathrm{~A} \mathrm{~g}^{-1}$ ) to be used in an electrochemical energy storage device with high power performance, and $5.0 \mathrm{~V} \_10 \mathrm{~h}$ electrode $\left(290.5 \mathrm{mAh} \mathrm{g}^{-1}\right.$ at $\left.50 \mathrm{~mA} \mathrm{~g}^{-1}\right)$ to be used under the demand of high energy density.

\subsection{Effect of oxidation potentials on polarization and diffusion dynamic}

To understand the effect of potentiostatic oxidation on rate performance, impedance and diffusion coefficient measurements were performed. These measurements were restricted within two potential ranges, $2.7 \sim 1.8 \mathrm{~V}$ in cathodic process and $3.2 \sim 4.2 \mathrm{~V}$ in anodic process to ensure the electrodes being in faradic reaction-dominated potential region on the concern of their poor reversibility [1-4, 7]. After fitting the impedance spectra (Fig. S2) of the representative pristine $\mathrm{rGO}, 4.7 \mathrm{~V} \_10 \mathrm{~h}$ and $5.0 \mathrm{~V} \_10 \mathrm{~h}$ electrodes (an example fitted by our equivalent circuit model is shown Fig. S3), we obtain the potential dependent resistances of charge transfer $\left(\mathrm{R}_{\mathrm{CT})}\right.$ and solid-electrolyte interphase $\left(\mathrm{R}_{\mathrm{SEI}}\right)$ as illustrated in Fig. 4. Under all

potentials applied, pristine rGO electrode exhibits the highest $\mathrm{R}_{\mathrm{CT}}$ and lowest $\mathrm{R}_{\mathrm{SEI}}$, while 4.7V_10h electrode shows the lowest $\mathrm{R}_{\mathrm{CT}}$ and highest $\mathrm{R}_{\mathrm{SEI}}$. It is noteworthy that the averaged sum of $\mathrm{R}_{\mathrm{CT}}$ and $\mathrm{R}_{\mathrm{SEI}}\left(\mathrm{R}_{\mathrm{CT}+\mathrm{SEI}}\right)$ increases in the order of $4.7 \mathrm{~V}_{-} 10 \mathrm{~h}$ (336.2 Ohm), pristine (346.5 Ohm), 5.0V_10h $(387.6 \mathrm{Ohm})$. This result implies that moderate oxidations around 4.7 $\mathrm{V}$ can reduce the electrochemical polarization, but excessive oxidations above $4.9 \mathrm{~V}$ will deteriorate the electrochemical dynamic.

To investigate the ohmic polarization, IR drop was measured from galvanostatic charge-discharge curves (see Fig. S4 for the details of IR drop measurement). The linear dependence of IR drops on current densities is shown in Fig. 4(a) and these points were fitted by least-square linear method to give out the slopes (Fig. 4(b)). The slopes are determined by 
the electronic resistances of electrodes plus ionic resistance of electrolyte [46, 47]. Fig. 4(b) indicates that the resistance of rGO electrode decreases dramatically after the $4.6 \mathrm{~V}$ oxidation, then increases monotonously with enhancing potentials. The resistance decrease by $4.6 \mathrm{~V}$ oxidation is a result of the improved wettability of electrode in the electrolyte by potentialstatic oxidations. While the resistance increase by $5.0 \mathrm{~V}$ oxidation is due to the excessive disruption of $\pi$ conjugated network in rGO sheets.

Fig. 5 shows the lithium ion diffusion coefficients at various potentials, calculated by impedance spectra (Fig. S2) combining with GITT curves (Fig. S5). The averaged diffusion coefficient of $4.7 \mathrm{~V} \_10 \mathrm{~h}$ electrode $\left(2.3 \times 10^{-11} \mathrm{~cm}^{2} \mathrm{~s}^{-1}\right)$ is slightly higher than that of pristine electrode $\left(2.1 \times 10^{-11} \mathrm{~cm}^{2} \mathrm{~s}^{-1}\right)$, while $5.0 \mathrm{~V} \_10 \mathrm{~h}$ electrode has a lower value $\left(5.3 \times 10^{-12} \mathrm{~cm}^{2}\right.$ $\mathrm{s}^{-1}$ ). This result reveals that the transport of lithium ion in graphene sheets will be hindered by the excessive OCFGs because the interaction of lithium ion with OCFGs elevates the energy barrier for lithium ion bulk diffusion [48-50]. The slight increase for 4.7V_10h electrode can be interpreted by considering that the enlarged interlayer spacing lowers the activation energy for diffusion, however this effect is limited.

The measured impedance values and diffusion coefficients of the three representative electrodes indicate that the potentiostatic oxidations under moderate potentials minimizes the electrochemical and ohmic polarizations of rGO electrode without retarding diffusion dynamic, thereby enhancing rate performance. In combination with the high specific capacity induced by newly formed OCFGs, our potentiostatic oxidation approach is demonstrated to be effective for improving the pseudocapacitive performance of graphene electrode.

\section{Conclusions}

Tuning of the OCFGs of rGO has been achieved by potentiostatic oxidations in an EC/DMC carbonate-based electrolyte. Taking advantage of good bulk-sensitivity of TFY-XAS and XRD, we confirm that potentiostatic oxidations generate new electrochemically active OCFGs (i.e. $\mathrm{C}-\mathrm{O}-\mathrm{C},>\mathrm{C}=\mathrm{O}$ and $\mathrm{COOH}$ ) not only on outmost surface 
but also in the inner-layers of graphene sheets. This increasing of OCFGs in quantity contributes to the enhanced pseudocapacitive capacity. For instance, the specific capacity of 170.4 $\mathrm{mAh} \mathrm{g}^{-1}$ for pristine electrode is increased to $290.5 \mathrm{mAh} \mathrm{g}^{-1}$ after $5.0 \mathrm{~V}$ oxidation. Moreover, the potentiostatic oxidations at moderate potentials around $4.7 \mathrm{~V}$ can reduce the electrochemical and ohmic polarization, and makes the oxidized electrodes inherit the good diffusion dynamic, thereby improving the rate capability.

\section{Acknowledgements}

This work was supported by the National Natural Science Foundation of China (Grant nos. 51272051， 50872026). The Advanced Light Source is supported by the Director, Office of Science, Office of Basic Energy Sciences, of the U.S. Department of Energy under Contract No. DE-AC02-05CH11231. We thank Prof. Miquel Salmeron and Dr. Cheng Hao $\mathrm{Wu}$ at LBNL for the beam-time and manuscript revision. H.W. and B.F. acknowledge the support of Short-term Visiting Program from Harbin Institute of Technology for visiting at LBNL.Y.Y. Thanks the support of ALS Doctoral Fellowship.

\section{References}

[1] S.W. Lee, N. Yabuuchi, B.M. Gallant, S. Chen, B.S. Kim, P.T. Hammond, Y. Shao-Horn, Nat. Nanotechnol., 5 (2010) 531-537.

[2] S.W. Lee, B.M. Gallant, H.R. Byon, P.T. Hammond, Y. Shao-Horn, Energy Environ. Sci., 4 (2011) 1972-1985.

[3] S.W. Lee, B.M. Gallant, Y. Lee, N. Yoshida, D.Y. Kim, Y. Yamada, S. Noda, A. Yamada, Y. Shao-Horn, Energy Environ. Sci., 5 (2012) 5437-5444.

[4] S.Y. Kim, J. Hong, R. Kavian, S.W. Lee, M.N. Hyder, Y. Shao-Horn, P.T. Hammond, Energy Environ. Sci., 6 (2013) 888-897.

[5] B.M. Gallant, S.W. Lee, T. Kawaguchi, P.T. Hammond, Y. Shao-Horn, J. Electrochem. Soc., 161 (2014) A1625-A1633.

[6] T.Y. Liu, A.A.B. Davijani, J.Y. Sun, S. Chen, S. Kumar, S.W. Lee, Small, 12 (2016) 3423-3431.

[7] B.Z. Jang, C. Liu, D. Neff, Z. Yu, M.C. Wang, W. Xiong, A. Zhamu, Nano Lett., 11 (2011) 3785-3791.

[8] A. Zhamu, G.R. Chen, C.G. Liu, D. Neff, Q. Fang, Z.N. Yu, W. Xiong, Y.B. Wang, X.Q. Wang, B.Z. Jang, Energy Environ. Sci., 5 (2012) 5701-5707. 
[9] D.-W. Wang, C. Sun, G. Zhou, F. Li, L. Wen, B.C. Donose, G.Q. Lu, H.-M. Cheng, I.R. Gentle, J. Mater. Chem. A, 1 (2013) 3607-3612.

[10] S.H. Ha, Y.S. Jeong, Y.J. Lee, ACS Appl Mater Interfaces, 5 (2013) 12295-12303.

[11] H. Kim, H.D. Lim, S.W. Kim, J. Hong, D.H. Seo, D.C. Kim, S. Jeon, S. Park, K. Kang, Sci. Rep., 3 (2013) 1506.

[12] Z. Weng, F. Li, D.W. Wang, L. Wen, H.M. Cheng, Angew. Chem. Int. Ed. Engl., 52 (2013) 3722-3725.

[13] H. Kim, K.Y. Park, J. Hong, K. Kang, Sci. Rep., 4 (2014) 5278.

[14] W. Ai, Z.Z. Du, Z.X. Fan, J. Jiang, Y.L. Wang, H. Zhang, L.H. Xie, W. Huang, T. Yu, Carbon, 76 (2014) 148-154.

[15] D.B. Xiong, X.F. Li, H. Shan, B. Yan, L.T. Dong, Y. Cao, D.J. Li, J. Mater. Chem. A, 3 (2015) 11376-11386.

[16] T.Y. Liu, K.C. Kim, R. Kavian, S.S. Jang, S.W. Lee, Chem. Mater., 27 (2015) 3291-3298.

[17] D.B. Xiong, X.F. Li, H. Shan, Y. Zhao, L. Dong, H. Xu, X.F. Zhang, D.J. Li, X.L. Sun, Electrochim. Acta, 174 (2015) 762-769.

[18] X.Y. Shan, Y.Z. Wang, D.W. Wang, F. Li, H.M. Cheng, Adv. Energy Mater., 6 (2016) 1502064.

[19] D.B. Xiong, X.F. Li, H. Shan, B. Yan, D.J. Li, C. Langford, X.L. Sun, Appl. Energy, 175 (2016) 512-521.

[20] H.R. Byon, B.M. Gallant, S.W. Lee, Y. Shao-Horn, Adv. Funct. Mater., 23 (2013) 1037-1045.

[21] B. Lee, C. Lee, T.Y. Liu, K. Eom, Z.M. Chen, S. Noda, T.F. Fuller, H.D. Jang, S.W. Lee, Nanoscale, 8 (2016) 12330-12338.

[22] T.Y. Liu, R. Kavian, Z.M. Chen, S.S. Cruz, S. Noda, S.W. Lee, Nanoscale, 8 (2016) 3671-3677.

[23] H.L. Guo, X.F. Wang, Q.Y. Qian, F.B. Wang, X.H. Xia, ACS Nano, 3 (2009) 2653-2659.

[24] M.D. Levi, D. Aurbach, J. Phys. Chem. B, 101 (1997) 4641-4647.

[25] C. Ho, I.D. Raistrick, R.A. Huggins, J. Electrochem. Soc., 127 (1980) 343-350.

[26] S.J. Sabounchei, M. Panahimehr, H. Keypour, M.H. Zebarjadian, J. Mol. Struct., 1040 (2013) 184-191.

[27] F. Zhao, M. Zhan, W.X. Zhang, Z.F. Xi, Organometallics, 32 (2013) 2059-2068.

[28] J.G. Zhou, H.T. Fang, J.M. Maley, J.Y.P. Ko, M. Murphy, Y. Chu, R. Sammynaiken, T.K. Sham, J. Phys. Chem. C, 113 (2009) 6114-6117.

[29] B.J. Schultz, C.J. Patridge, V. Lee, C. Jaye, P.S. Lysaght, C. Smith, J. Barnett, D.A. Fischer, D. Prendergast, S. Banerjee, Nat. Commun., 2 (2011) 372.

[30] J.J. Velasco-Velez, C.H. Chuang, H.L. Han, I. Martin-Fernandez, C. Martinez, W.F. Pong, Y.R. Shen, F. Wang, Y. Zhang, J. Guo, M. Salmeron, J. Electrochem. Soc., 160 (2013) C445-C450.

[31] B.J. Schultz, R.V. Dennis, J.P. Aldinger, C. Jaye, X. Wang, D.A. Fischer, A.N. Cartwright, S. Banerjee, RSC Adv., 4 (2014) 634-644.

[32] R. Qiao, I.T. Lucas, A. Karim, J. Syzdek, X. Liu, W. Chen, K. Persson, R. Kostecki, W. 
Yang, Adv. Mater. Interfaces, 1 (2014) 1300115.

[33] L. Cheng, E.J. Crumlin, W. Chen, R. Qiao, H. Hou, S. Franz Lux, V. Zorba, R. Russo, R. Kostecki, Z. Liu, K. Persson, W. Yang, J. Cabana, T. Richardson, G. Chen, M. Doeff, Phys. Chem. Chem. Phys., 16 (2014) 18294-18300.

[34] C. Yogi, D. Takamatsu, K. Yamanaka, H. Arai, Y. Uchimoto, K. Kojima, I. Watanabe, T. Ohta, Z. Ogumi, J. Power Sources, 248 (2014) 994-999.

[35] A. Ganguly, S. Sharma, P. Papakonstantinou, J. Hamilton, J. Phys. Chem. C, 115 (2011) 17009-17019.

[36] H. Xu, X. Fan, Y. Lu, L. Zhong, X. Kong, J. Wang, Carbon, 48 (2010) 3300-3303.

[37] D. Ye, Y. Yu, J. Tang, L. Liu, Y. Wu, Nanoscale, 8 (2016) 10406-10414.

[38] J. Lu, J.X. Yang, J.Z. Wang, A.L. Lim, S. Wang, K.P. Loh, Acs Nano, 3 (2009) 2367-2375.

[39] M. Mao, M. Wang, J. Hu, G. Lei, S. Chen, H. Liu, Chem. Commun., 49 (2013) 5301-5304.

[40] T. Ishihara, Y. Yokoyama, F. Kozono, H. Hayashi, J. Power Sources, 196 (2011) 6956-6959.

[41] J.A. Seel, J.R. Dahn, J. Electrochem. Soc., 147 (2000) 892-898.

[42] L.J. Hardwick, M. Hahn, P. Ruch, M. Holzapfel, W. Scheifele, H. Buqa, F. Krumeich, P. Novak, R. Kotz, Electrochim. Acta, 52 (2006) 675-680.

[43] L.J. Hardwick, P.W. Ruch, M. Hahn, W. Scheifele, R. Kötz, P. Novák, J. Phys. Chem. Solids, 69 (2008) 1232-1237.

[44] H. Nakagawa, Y. Domi, T. Doi, M. Ochida, S. Tsubouchi, T. Yamanaka, T. Abe, Z. Ogumi, J. Power Sources, 206 (2012) 320-324.

[45] M.E. Stournara, V.B. Shenoy, J. Power Sources, 196 (2011) 5697-5703.

[46] Z. Lei, N. Christov, X.S. Zhao, Energy Environ. Sci., 4 (2011) 1866.

[47] A.G. Pandolfo, A.F. Hollenkamp, J. Power Sources, 157 (2006) 11-27.

[48] K. Persson, V.A. Sethuraman, L.J. Hardwick, Y. Hinuma, Y.S. Meng, A. van der Ven, V. Srinivasan, R. Kostecki, G. Ceder, J. Phys. Chem. Lett., 1 (2010) 1176-1180.

[49] K. Persson, Y. Hinuma, Y.S. Meng, A. Van der Ven, G. Ceder, Phys. Rev. B, 82 (2010) 125416.

[50] L. Mandeltort, J.T. Yates, J. Phys. Chem. C, 116 (2012) 24962-24967.

\section{Figures}



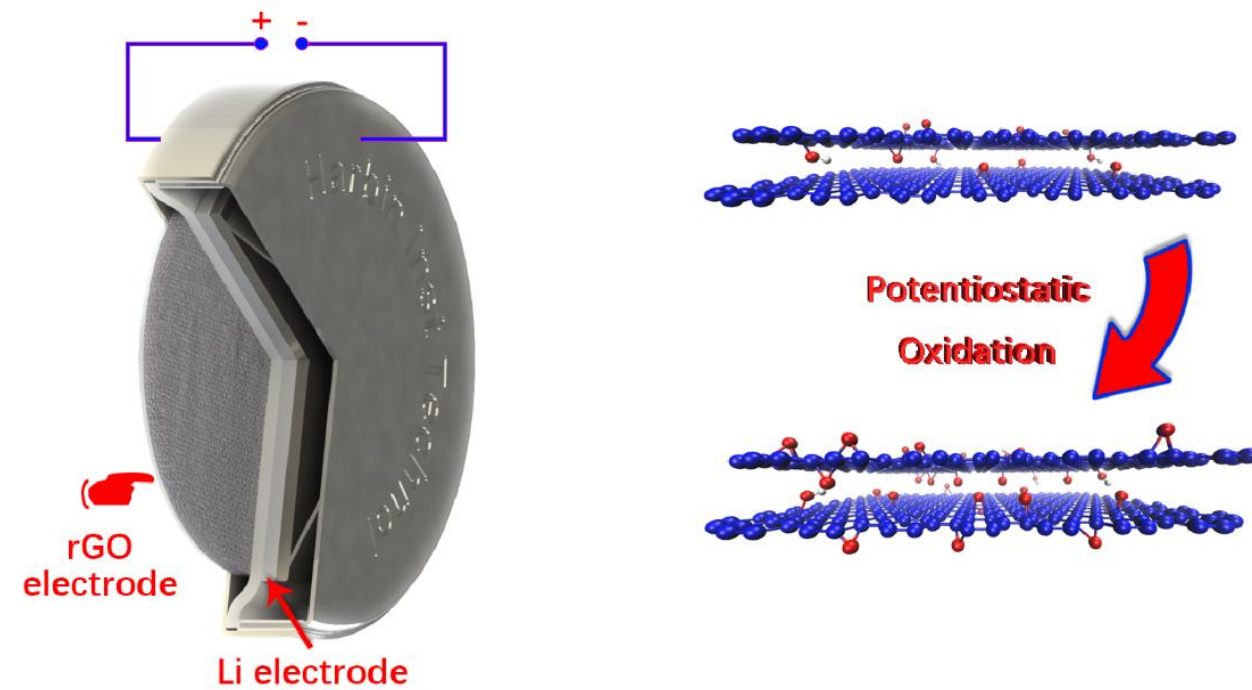

\section{Potentiostatic}

Oxidation

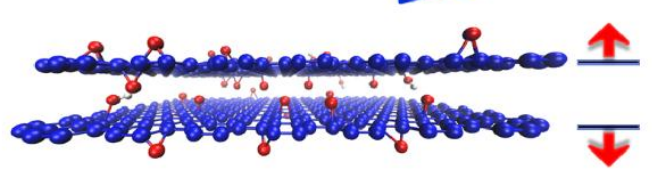

Li electrode

Fig. 1. Schematic illustration of the potentiostatic oxidation process, and the chemical and structural change of rGO (only bi-layer structure is drawn for simplification). Blue and red ball stand for $\mathrm{C}$ and $\mathrm{O}$ atoms, respectively.

(a)

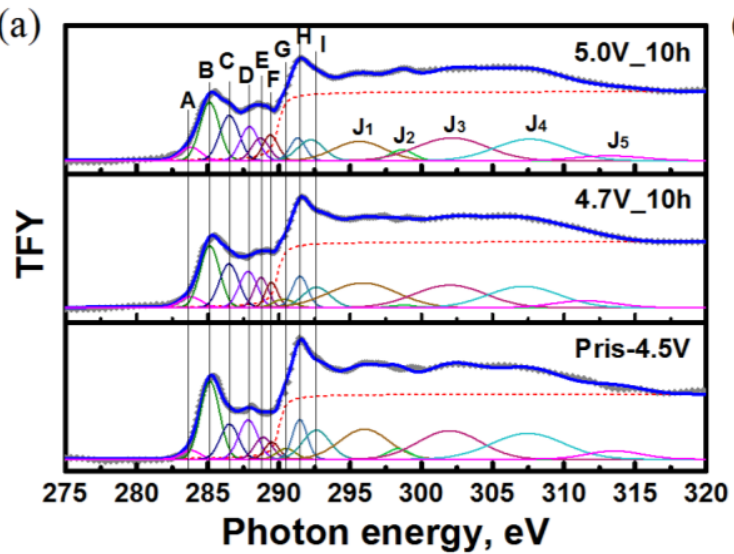

(c)

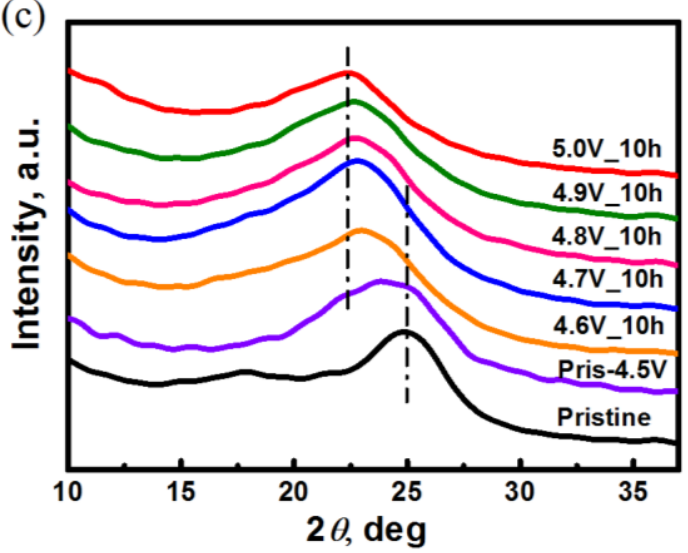

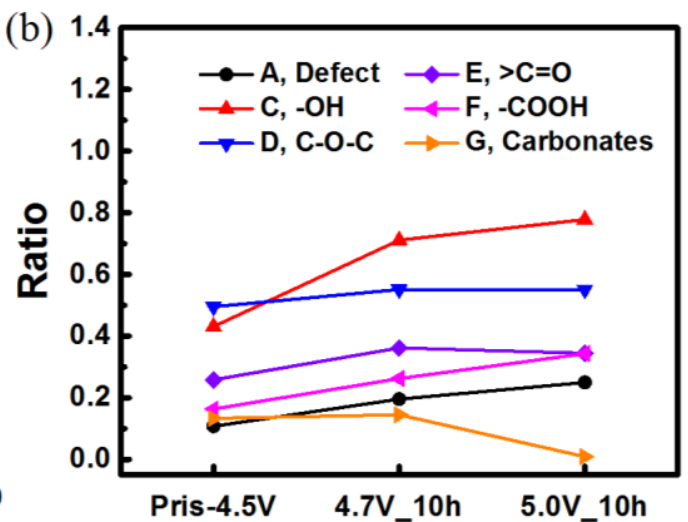

(d)

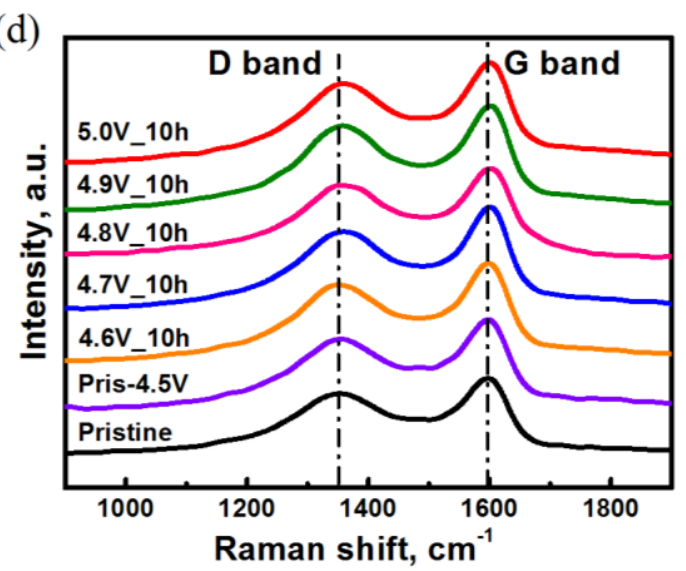

Fig. 2. (a) C K-edge XAS spectra of pristine rGO, 4.7V_10h and 5.0V_10h electrodes, (b) 
area ratios of OCFG peaks with $\mathrm{C} \pi^{*}$ resonance peak for the pristine and oxidized rGOs. (c) XRD patterns and (d) Raman spectra of pristine and oxidized rGOs.

(a)

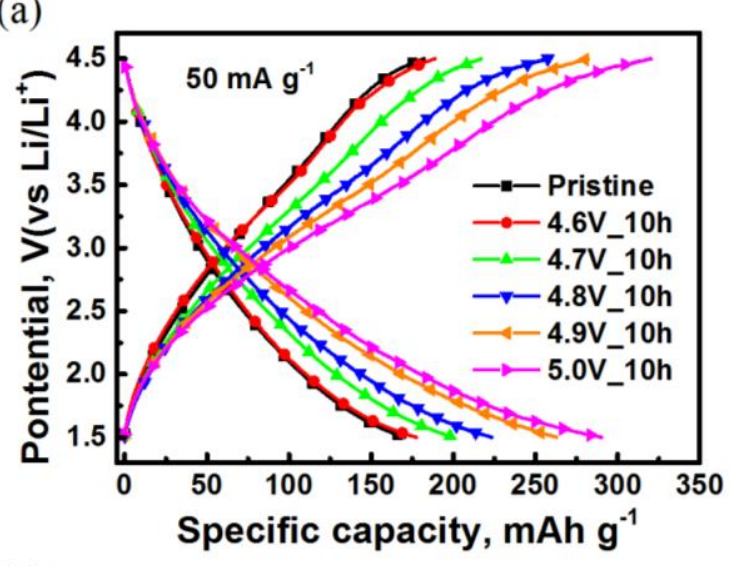

(c)

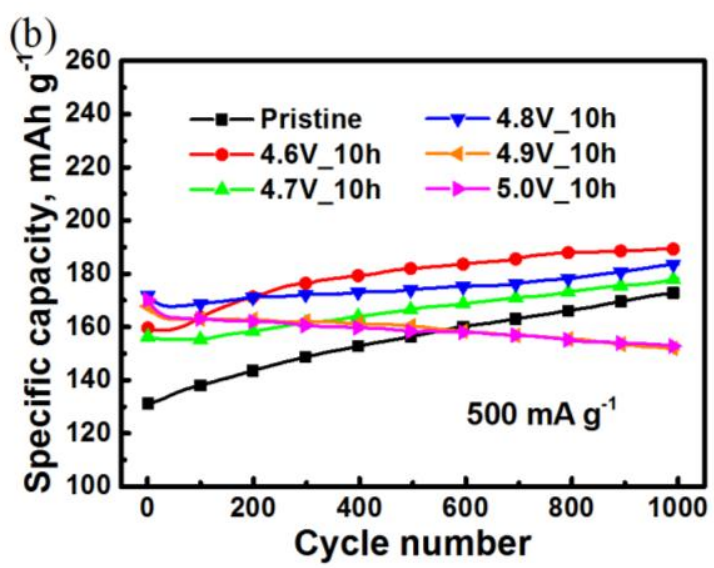

Normalized capacity (\%)

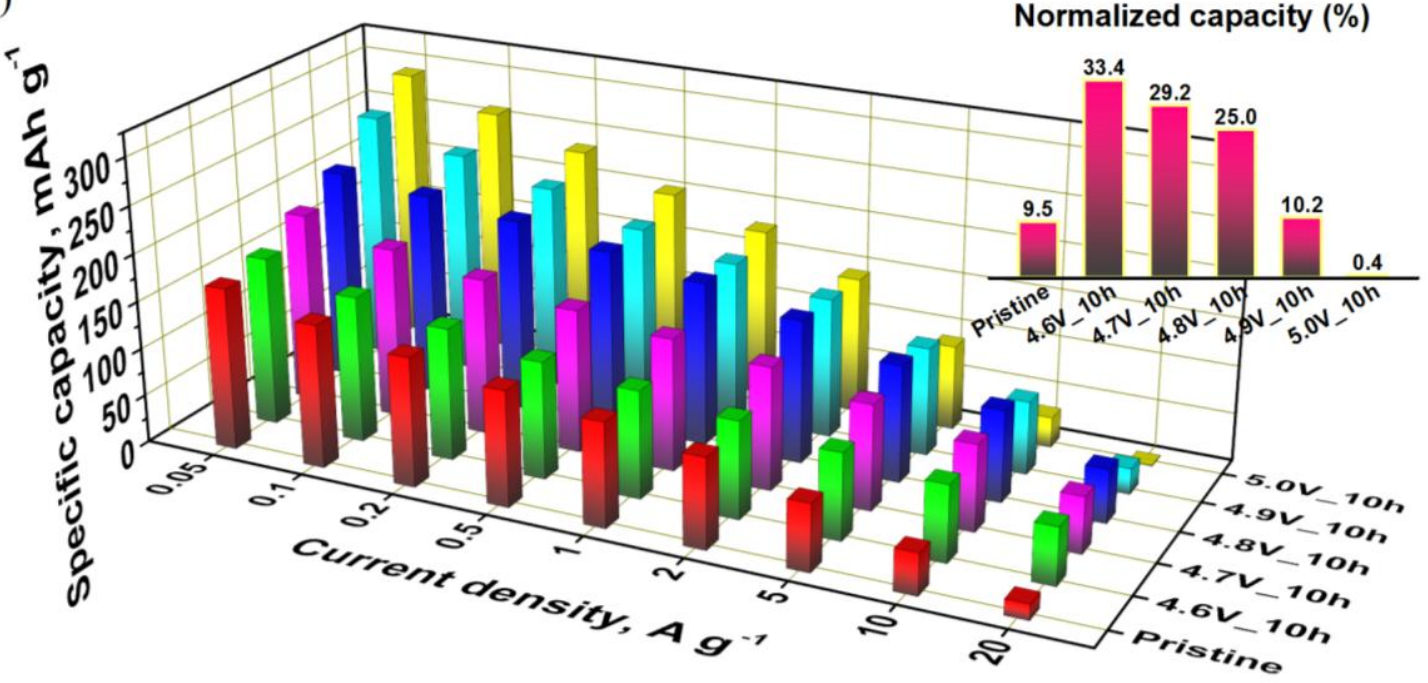

Fig. 3. Electrochemical performance of pristine and oxidized rGO electrodes. (a) charge-discharge curves under a current density of $50 \mathrm{~mA} \mathrm{~g}^{-1}$, (b) cycle performance of pristine and oxidized rGO electrodes at $0.5 \mathrm{~A} \mathrm{~g} \mathrm{~g}^{-1}$. (c) discharge specific capacities at the current densities from $50 \mathrm{~mA} \mathrm{~g}^{-1}$ to $20 \mathrm{~A} \mathrm{~g}^{-1}$. The insert in (c) is normalized capacities at $20 \mathrm{~A}$ $\mathrm{g}^{-1}$ with respective to those at $50 \mathrm{~mA} \mathrm{~g}^{-1}$ for each electrode. 

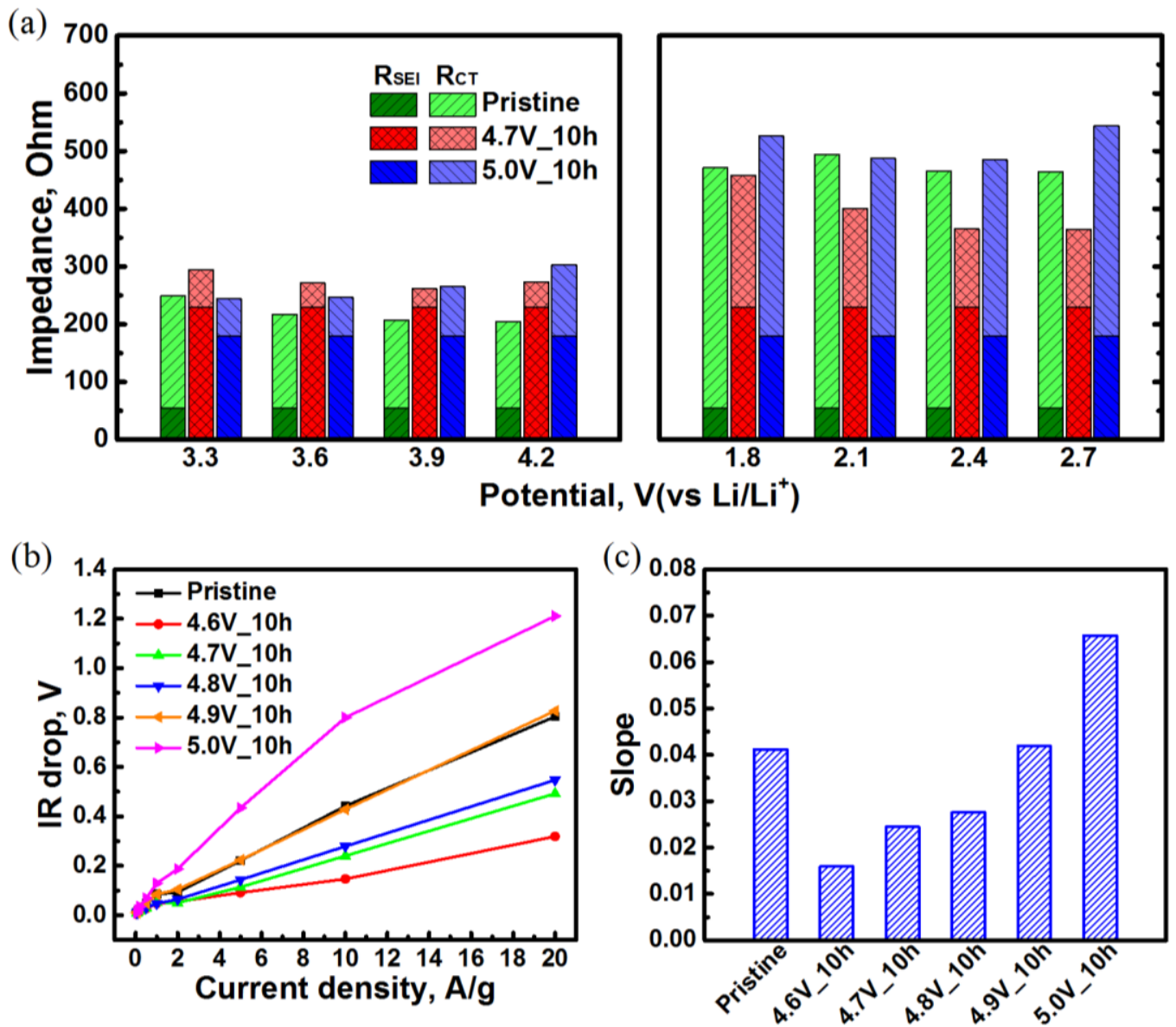

Fig. 4. (a) Potential-dependent $\mathrm{R}_{\mathrm{CT}}$ and $\mathrm{R}_{\mathrm{SEI}}$ of pristine rGO, 4.7V_10h and 5.0V_10h electrodes in faradic reaction-dominated potential region. Left and right panels are recorded in anodic and cathodic polarization processes, respectively. (b) Dependence of IR drops on current densities, (c) slopes of IR drops vs current densities for pristine rGO and oxidized rGO. 


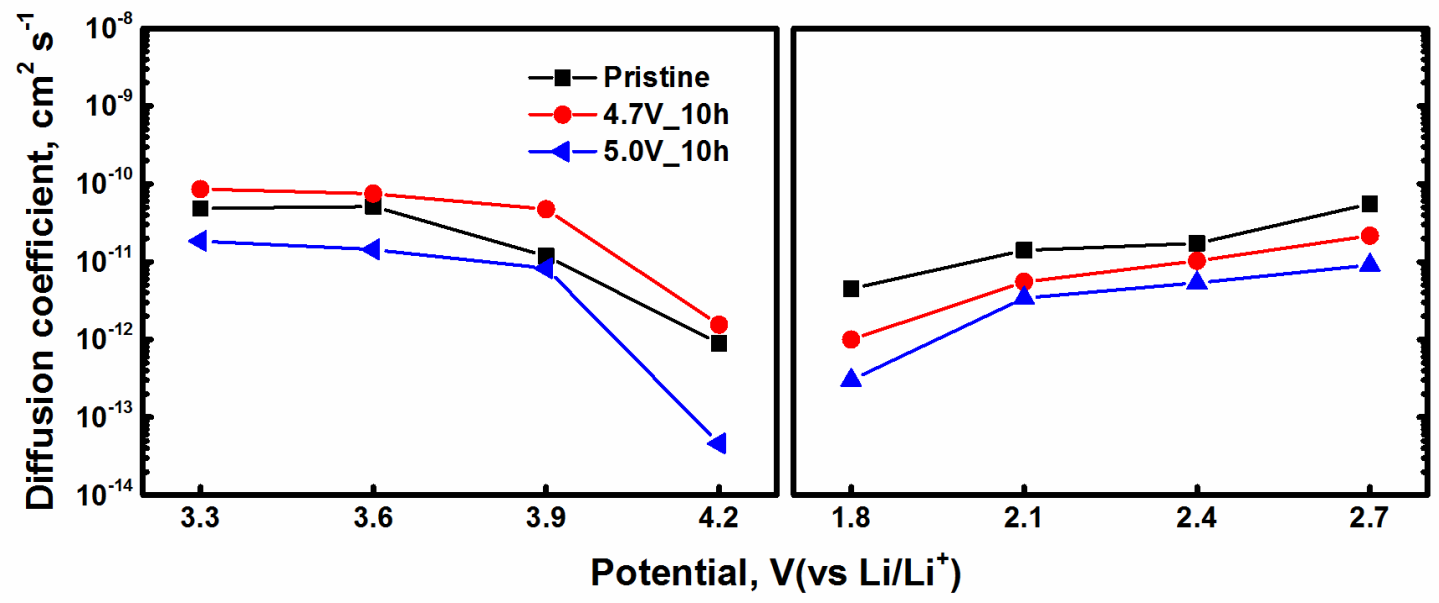

Fig. 5. Potential-dependent lithium ion diffusion coefficients of pristine rGO, $4.7 \mathrm{~V} \_10 \mathrm{~h}$ and 5.0V_10 electrodes in anodic (from 3.3 to $4.2 \mathrm{~V}$ ) and cathodic (from 2.7 to $1.8 \mathrm{~V}$ ) reaction-dominated potential regions. 


\section{Graphical Abstract}

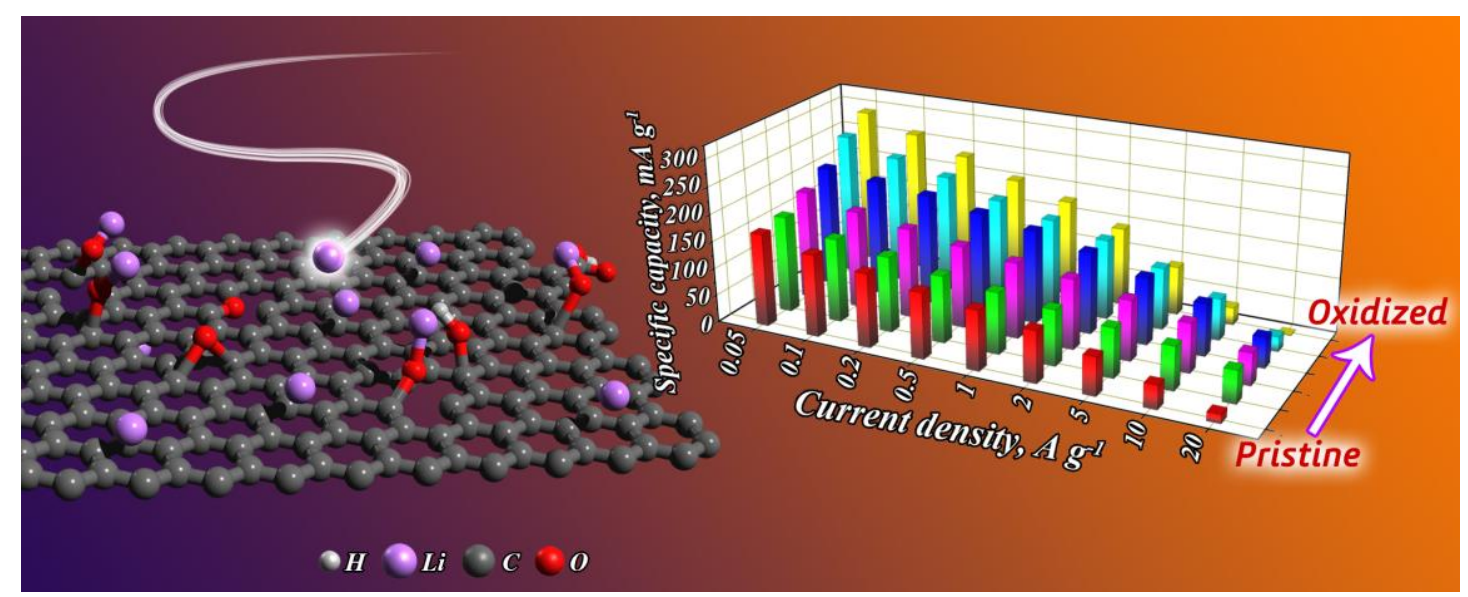

Tuning inner-layer oxygen functional groups of reduced graphene oxide by potentiostatic oxidation in carbonate-based electrolyte improves the electrochemical performance. 